\title{
Integración regional andina en salud
}

\author{
Health in Andean regional integration
}

\author{
${ }^{1}$ Facultad de Medicina, \\ Universidad Nacional de \\ Colombia, Bogotá, Colombia. \\ 2 Red Andina y del Caribe de \\ Investigación en Políticas $y$ \\ Sistemas de Salud, Bogotá, \\ Colombia. \\ Correspondencia \\ C. A. Agudelo \\ Instituto de Salud Pública. \\ Facultad de Medicina, \\ Universidad Nacional de \\ Colombia. \\ Ciudad Universitaria \\ Edificio 471, Bogotá, \\ Colombia. \\ caagudeloc@unal.edu.co
}

\begin{abstract}
Despite their shared history, the Andean countries are socially and politically diverse, with heterogeneous health realities and complex integration processes. General developments such as the Latin American Free Trade Association and Latin American Integration Association have existed for decades, along with others of a regional scope, like the Andean Community of Nations, Caribbean Community, and Central American Common Market. The health field has a specific instrument in the Andean Region called the Hipólito Unánue Agreement, created in 1971. Integration processes have concentrated on economic aspects, based on preferential customs agreements that have led to an important long-term increase in trade. Less progress has been made in the field of health in terms of sharing national experiences, knowledge, and capabilities. Analysis of experiences in health has shown that integration depends on the countries' respective strengths and to a major extent on national political processes.
\end{abstract}

Health Services; Health Policy; Health Systems
Carlos A. Agudelo 1,2

\section{Introducción}

La integración en salud de los países de la región andina forma parte de los procesos generales de integración que se dan entre ellos. Ambos están condicionados por múltiples factores del contexto global y regional cuya relación suele ser compleja. Para aproximarnos al examen de la integración en salud entre estos países consideramos, en primer lugar, cómo operan algunos de los condicionantes y luego valoramos el proceso regional.

\section{Contexto global y regional}

Desde 1990 el mundo ha entrado en una nueva fase de organización y agrupamiento internacional, generada por el hundimiento de la Unión Soviética. La política y la situación internacional han cambiado progresivamente de signo, a medida que el mundo abandona el esquema bipolar y adopta el esquema unipolar de recomposición de las fuerzas internacionales y regionales 1 .

En la globalización, como nueva fase histórica de la época moderna, se han presentado al menos de tres grandes campos de transformaciones y reagrupamientos: (1) intensificación de tendencias previas en la expansión de la producción y los mercados capitalistas; (2) nuevo marco cualitativo de ordenamiento bajo la hegemonía del capital financiero internacional; y (3) recon- 
formación de las relaciones y de la interacción entre economía, sociedad, política, cultura y comunicación.

A pesar de que la configuración del mapa geopolítico internacional no ha concluido, es hoy suficientemente claro y permite delimitar algunas características claves de las fases históricas precedentes y actuales, como se indican en las siguientes características geopolíticas:

- Fase 1945-1990: mundo bipolar, equilibrio nuclear estratégico, multipolaridad política, rivalidad intercapitalista, corporaciones, y transnacionales.

- Fase 1991 en adelante: mundo unipolar, monopolio nuclear, multipolaridad de bloques, fusión corporativa, supranacional, y corporaciones globales.

En este marco de transformaciones, la economía internacional presenta unas tendencias marcadas hacia el mercantilismo, la liberalización económica asimétrica y la integración de mercados. De otro lado, se perciben cambios estructurales en los sistemas productivos (producción y organización flexibles, tipos y formas de acumulación). Probablemente, los cambios más evidentes son los que han experimentado los modelos de desarrollo: predominio neoliberal, políticas de ajuste, reestructuración de instituciones, de lo público y lo social, y los sistemas colectivos de preferencias culturales 1,2. De manera más visible que en la fase previa, ahora surgen sistemas funcionales de agrupamientos de países, en términos de sostenibilidad económica y políticas nacionales e inserción en los esquemas de hegemonía internacional.

Con respecto a los países del Norte, son reconocibles algunas combinaciones entre Estados 3 que se apoyan en herramientas de articulación y regulación internacional. En seguridad regional y global: la Organización del Tratado del Atlántico Norte, y los frentes militares de escarmiento organizados por la Organización de las Naciones Unidas (ONU). En el campo económico, Estados Unidos, Japón, Europa, el G7 y su incidencia en la Organización Mundial del Comercio (OMC). La alianza Estados Unidos y Japón proyectada sobre el comercio mundial y la seguridad asiática; Europa en el campo comercial y las finanzas mundiales; la alianza Rusia y Estados Unidos, en pos del desarme nuclear. Por otra parte, juegan un papel significativo los acuerdos de la OMC, como los Derechos de Propiedad Intelectual relacionados con el comercio y el Acuerdo sobre el Comercio de Servicios, o los más directamente relacionados con la salud y las políticas sanitarias (acuerdo sobre obstáculos técnicos al comercio, acuerdo sobre la aplicación de medidas sanitarias y fitosanitarias).
De otra parte, los países del Sur se orientan débilmente a un reconcentramiento en torno a potencias medias, como África del Sur, Siria, India y Brasil.

\section{Región Andina}

Pese a que los países de la región andina - Venezuela, Colombia, Ecuador, Perú y Bolivia - tienen una historia en común y unos sistemas políticos similares, presentan también importantes diferencias en cuanto a las formas de inserción en la política y la economía internacional, su grado de desarrollo, sus tendencias epidemiológicas y su situación de salud. De esta historia común y de esta diversidad social se desprenden realidades políticas y sanitarias heterogéneas y procesos de integración complejos. Algunas de estas características y diferencias pueden ser ilustradas, como se indica en la Tabla $14,5,6$.

\section{Diversidad en los sistemas de salud}

Un referente crucial de la integración andina está en directa relación con los sistemas nacionales de salud, cuya diversidad debe destacarse. El análisis comparado de esta diversidad permite agrupar los países por medio de unas categorías claves 5,6,7,8,9,10,11,12,13,14,15.

Con base en el criterio de fuentes de financiamiento, los sistemas de salud de los países andinos conforman dos grupos: aquellos que combinan cotizaciones y aportes del Estado, como Colombia y Ecuador, y los que utilizan principalmente las cotizaciones, como Bolivia, Perú y Venezuela. La cobertura no es universal en ninguno de los países andinos considerados. Hacia los años 2000-2003, la cobertura en servicios era de $66 \%$ en Bolivia, $73 \%$ en Ecuador, $71 \%$ en Perú y de más del $90 \%$ en Colombia, pero con un nivel de aseguramiento de sólo 54\%. Por su grado de unidad, se consideran segmentados sin coordinación los sistemas de salud de Bolivia, Ecuador, Perú y Venezuela; y coordinado sin integración, el sistema de Colombia. En cuanto a la separación de funciones - financiamiento, aseguramiento, provisión, supervisión -, Colombia la ha puesto en práctica y Perú lo ha hecho parcialmente. Pero, Bolivia, Ecuador y Venezuela no han introducido la separación de funciones. Por último, Colombia y Bolivia tienen un alto grado de descentralización en salud, mientras que Ecuador, Perú y Venezuela presentan un grado bajo.

Esta diversidad de sistemas de salud, ligada a los caminos de desarrollo que ha escogido cada país, introduce dificultades para avanzar en procesos de integración en las políticas y sistemas de salud, y para visualizar los efectos positivos y 
Indicadores sociodemográficos y sanitarios. Países andinos.

\begin{tabular}{|c|c|c|c|c|c|c|}
\hline Indicadores & Año & Bolivia & Colombia & Ecuador & Perú & Venezuela \\
\hline Población total (en miles) & 2003 & 8.808 & 44.222 & 13.003 & 27.167 & 25.699 \\
\hline Tasa cruda de natalidad (por 1.000 habitantes) & 2003 & 28,7 & 21,9 & 22,6 & 22,9 & 22,5 \\
\hline Tasa cruda de mortalidad (por 1.000 habitantes) & 2003 & 8,0 & 5,4 & 5,8 & 6,1 & 4,6 \\
\hline Crecimiento demográfico anual (\%) & 2003 & 1,9 & 1,6 & 1,5 & 1,5 & 1,8 \\
\hline Tasa global de fecundidad (hijos/mujer) & 2003 & 3,7 & 2,6 & 2,7 & 2,8 & 2,7 \\
\hline Población urbana (\%) & 2003 & 64,0 & 76,5 & 64,3 & 73,9 & 87,6 \\
\hline Esperanza de vida al nacer (en años) & 2004 & 64 & 72 & 71 & 70 & 74 \\
\hline Tasa de mortalidad materna (por 100.000 nacidos vivos/año) & 2003 & 230 & 99 & 81 & 185 & 68 \\
\hline Tasa de mortalidad infantil (por 1.000 nacidos vivos) & 2003 & 56 & 18 & 24 & 26 & 18 \\
\hline PIB per capita total (US\$) & 2001 & 125 & 356 & 177 & 231 & 386 \\
\hline Gasto en salud como \% del PIB & 2002 & 5,5 & 9,3 & 4,0 & 4,4 & 8,8 \\
\hline Gasto público en salud/gasto sanitario total (\%) & 2001 & 66,3 & 65,7 & 50,3 & 55,0 & 62,1 \\
\hline
\end{tabular}

negativos de las diferentes opciones. En realidad, los procesos de integración no han incluido los sistemas de salud y, por tanto, no los han puesto a prueba, en una perspectiva de mejorar su cobertura y equidad. Es más, la capacidad de respuesta y de adaptación de los sistemas de salud en los procesos de integración no ha sido adecuadamente estudiada.

\section{Procesos y alcances de la integración andina}

En el marco de las tendencias descritas se han construido procesos limitados de integración regional en América Latina, que representan, por tanto, un doble papel: acomodamiento a la globalización y perspectiva de conservación nacional.

A manera de reseña, los procesos de alcance general son los siguientes: Asociación Latinoamericana de Libre Comercio (ALALC - 1960), Asociación Latinoamericana de Integración (ALADI - conformada por Argentina, Bolivia, Brasil, Chile, Colombia, Ecuador, Paraguay, Perú, México, Uruguay y Venezuela en 1980), Asociación de Libre Comercio de las Américas (ALCA) y la Comunidad Suramericana de Naciones. Esta última tiene como áreas de acción prioritaria el diálogo político, la integración física, el medio ambiente, integración energética, promoción de la cohesión social, de la inclusión social y las telecomunicaciones.

Un apreciable número de iniciativas de integración regional y/o bilateral han tenido lugar desde décadas atrás. Cabe destacar el Pacto An- dino, la Comunidad Andina de Naciones (1969) y el Tratado Amazónico (1978). Adicionalmente, en 1998, se suscribió el Acuerdo Marco para la creación de una Zona de Libre Comercio entre la Comunidad Andina y el MERCOSUR.

Los procesos de integración andina se orientan en gran parte por la declaración de la Cumbre Presidencial Andina de 1999 (Comunidad Andina de Naciones. http://www.comunidadandina. org/documentos.asp, accedido el Jun/2006): “Decidimos que en el próximo lustro, las tareas prioritarias para profundizar nuestra integración serán el establecimiento de un Mercado Común, la instrumentación de la política exterior común, el desarrollo de una Agenda Social, la ejecución de una política comunitaria de integración y desarrollo fronterizo, la definición y ejecución de políticas de desarrollo sostenible y el fortalecimiento institucional".

Los procesos de integración de alcance regional son el Pacto Andino-Comunidad Andina de Naciones y el Sistema Andino de Integración, la Zona de Libre Comercio, el Tratado de Preferencias Arancelarias Andinas (ATPA) y el Mercado Común Andino. Más recientemente se ha negociado un Tratado de Libre Comercio (TLC), entre Colombia, Perú, Ecuador y Estados Unidos. De otro lado, se encuentra el Tratado Amazónico (Bolivia, Brasil, Colombia, Ecuador, Guyana, Perú, Suriname y Venezuela). Con alguna frecuencia se dan alianzas bilaterales entre los países de la región andina y de estos con países de otras regiones. 
Temáticas centrales en los procesos de integración regional

En el ámbito general, al estilo de la Comunidad Sudamericana de Naciones, predominan algunas temáticas: (a) económicas y comerciales, usualmente centradas en las preferencias arancelarias y las áreas de libre comercio, incluidos los mercados comunes; (b) la integración en los aspectos estatales y políticos: diálogo político, integración física, medio ambiente, integración energética, mecanismos financieros sudamericanos; (c) promoción de la cohesión social, de la inclusión y de la justicia social; y (d) las telecomunicaciones.

A pesar del alcance amplio con el que son planteados estos acuerdos, los desarrollos se han concentrado en los sistemas de preferencias arancelarias cuyo horizonte son las zonas de libre comercio.

En los ámbitos regionales predominan los aspectos de la integración con base en el comercio de bienes y servicios, las uniones aduaneras, la circulación de personas y los mercados comunes. Asimismo, se generan espacios de discusión y aproximación, en términos de la sostenibilidad de gobiernos democráticos, políticas exteriores comunes, desarrollo fronterizo y seguridad, políticas de erradicación de la pobreza, lucha contra las drogas y el terrorismo, desarrollo sostenible e integración económica.

A pesar de que se trata de instrumentos que tienen un alcance limitado, por medio de ellos se ha construido un comercio intracomunitario de relativa importancia. En la Comunidad Andina, la balanza comercial ha mostrado signos de recuperación a partir de 1998, a pesar de las dificultades económicas de algunos de los países miembros. Durante el periodo comprendido entre 1991 y el año 2000 la balanza comercial tuvo superávit, con excepción de 1998. Las importaciones pasaron de US\$30 millones en 1991 a US\$ 57 millones en el 2000, principalmente las procedentes de los países miembros del Tratado de Libre Comercio de América del Norte (TLCAN o NAFTA). Las exportaciones han presentado una tendencia al alza en este mismo periodo, resaltando las de orden intracomunitario que pasaron de US $\$ 1,8$ millones en 1991 a US\$ 5 millones en el 2000. Sin embargo, los principales destinos de estas exportaciones siguen siendo los países miembros del TLCAN y la Unión Europea (UE). En la última década, la composición de los destinos de las exportaciones ha cambiado: el comercio intracomunitario se ha acrecentado y ha aumentando el comercio con el principal socio, Estados Unidos. Las exportaciones hacia la UE han reducido su participación de manera importante al representar el $21 \%$ en 1991 y pasar al 11\% en el año 2000. En cuanto a las exportaciones intracomunitarias, Colombia fue el país con la más alta participación en la Comunidad en el año 2000, con ventas que alcanzaron los US\$ 2.161 millones. Cifra ésta muy por encima de los US\$ 1.612 millones correspondientes a sus importaciones en el mismo año. Bolivia fue, por el contrario, el país de la Comunidad Andina con menor participación en las exportaciones intracomunitarias, con unas ventas de US\$ 311 millones (Convenio Hipólito Unánue. http://intranet.comunidadandina.org/IDocumentos/c Newdocs.asp?GruDoc=13 accedido el Jul/2006). Estas tendencias se conservan después del año 2000.

De las importaciones totales en el año 2000, el 13.8\% (5.466 millones de US\$) correspondió a importaciones de orden intracomunitario. El país que realizó más operaciones de compra en la Comunidad fue Colombia (US\$ 1.612 millones), seguido por Venezuela (US\$1.439 millones) y Perú (US\$ 1.399 millones). Es de anotar que, aunque Bolivia fue el país de la Comunidad Andina con la mayor evolución en las importaciones, entre 1995 y el 2000, también fue el país que menos compró a los demás países comunitarios.

\section{Integración en salud}

El instrumento principal de integración en salud es el Convenio Hipólito Unánue. Es un convenio de cooperación en salud, creado en 1971 con la participación de Bolivia, Colombia, Chile, Ecuador, Perú y Venezuela. El Convenio fue adscrito al Sistema Andino de Integración en 1998. Para los años 2006-2007, el Convenio Hipólito Unánue se ha planteado cuatro áreas estratégicas con sus respectivas líneas de acción (http:/ / www.oras conhu.org/index.php?IDIOMA=SP\&planilha+co ntenido\&ncategorial=101, accedido el Jul/2006). - Salud en fronteras y vigilancia epidemiológica: Red Andina de Vigilancia Epidemiológica, Plan Andino de Salud en las Fronteras, Proyecto Control de la Malaria en las Zonas Fronterizas de la Región Andina: un Enfoque Comunitario, Salud Ambiental, Preparativos para Emergencias y Desastres;

- Políticas de medicamentos y tecnología en salud.

- Promoción y protección de la salud:VIH/Sida, lucha contra el tabaquismo, salud de los trabajadores, salud intercultural y salud visual;

- Educación y gestión: salud y educación, salud y economía, red andina hospitalaria, telecomunicación y salud.

En el marco del Convenio Hipólito Unánue se han llevado a cabo procesos que acercan los países y ponen en común sus experiencias, para 
compartir conocimientos obtenidos de la aplicación y evaluación de programas y estrategias.

\section{Un balance preliminar}

Los procesos de integración en la Región Andina son de vieja data. Si bien se han logrado progresos limitados en aspectos económicos de producción y comercio, aún resta un largo trecho para acercarse al estado de comunidad económica. En otros aspectos - laborales, servicios, fronteras, recursos humanos, etc. -, los avances han sido modestos y desiguales. Aunque los países expresan con frecuencia la voluntad de concertar esfuerzos para atacar problemas que comparten su origen territorial o sus determinantes sociales y culturales, la participación del sector salud en los procesos de integración ha sido marginal.

La integración en salud en la Región Andina tiene un alcance limitado y ha dejado de lado aspectos como el seguimiento de los sistemas nacionales de salud, la salud fronteriza y el acceso transfronterizo a servicios de salud, las agendas de investigación en salud y en integración regional, trabajo y recursos humanos. Es también notorio que no se ha generado capacidad para prever los impactos positivos y negativos de los tratados de libre comercio, la integración económica y en otros ámbitos, sobre la salud de las poblaciones, la salud pública, la equidad en el gasto y el acceso, las licencias y la producción de medicamentos, etc., y por ende, en la preparación de medidas atenuantes. Por tanto, no se ha generado capacidad anticipatoria para prever y afrontar los efectos indeseables de la integración en los sistemas y políticas de salud.

Periódicamente, los procesos de integración entran en crisis debido a los desequilibrios comerciales, los acuerdos bilaterales o multilate- rales con otros bloques o países, o los conflictos políticos y económicos entre países fronterizos, aspectos que no son exclusivos de la Región Andina. Cabe mencionar las políticas proteccionistas y las devaluaciones que han practicado algunos países y afectan a sus vecinos (por ejemplo, Brasil en el gobierno de Cardoso frente a Argentina), los conflictos fronterizos en torno al desarrollo industrial y su impacto ambiental (Argentina y Uruguay) o los repetidos intentos y acuerdos bilaterales que llevan a cabo los países que forman parte de la Comunidad Andina de Naciones y el MERCOSUR con la UE, Estados Unidos y otros países (recuérdese el conflicto reciente en la Comunidad Andina de Naciones y el retiro de Venezuela, motivado, según lo expresan sus líderes, por el acuerdo de TLC de Colombia y Perú con Estados Unidos).

\section{Algunos desafíos}

La diversidad regional y la multiplicidad de procesos bilaterales, multilaterales, regionales y globales, generan dificultades para identificar la forma como incide la integración en la salud de las poblaciones. Aún en medio de estas dificultades, el seguimiento y análisis de los procesos de integración requieren tener en cuenta los aspectos estratégicos y los impactos en las políticas y sistemas de salud. La adquisición de capacidad para investigar los complejos problemas de las políticas y sistemas de salud y la construcción de agendas de investigación deben incluir los nuevos desafíos que está generando la integración económica, política y social de nuestros países: los efectos sobre la universalidad de la protección social, la equidad, el acceso, la cobertura, el costo, la calidad, el trabajo y los recursos humanos en salud, así como el acceso transfronterizo a los servicios de salud, entre otros.

\section{Resumen}

A pesar su historia compartida, los países de la Región Andina presentan una diversidad social y política que genera realidades sanitarias heterogéneas y procesos de integración complejos. Se han dado por décadas procesos generales, como la Asociación Latinoamericana de Libre Comercio y la Asociación Latinoamericana de Integración, o de alcance regional como la Comunidad Andina de Naciones, la Comunidad del Caribe y el Mercado Común Centroamericano. En el campo específico de la salud se cuenta con un instrumento en la Región Andina, el Convenio Hipólito Unánue, creado en 1971. Estos procesos de integración se han concentrado en los as- pectos económicos, con base en acuerdos de preferencias arancelarias, los cuales han generado, en el largo plazo, un intercambio comercial apreciable. En el campo de la salud se ha avanzado menos, en términos de procesos que ponen en común experiencias nacionales, conocimientos y capacidades. El análisis de las experiencias de integración en salud muestra que esta depende de las fortalezas de cada país y, en gran parte, de los procesos políticos nacionales.

Servicios de Salud; Política de Salud; Sistemas de Salud 


\section{Referencias}

1. Garay LJ. Globalización y crisis. ¿Hegemonía o corresponsabilidad? Bogotá: Tercer Mundo Editores; 1999 .

2. Cardona DC. Relaciones y política internacional. Análisis Político 1989; (8):354-9.

3. Socorro J, Cadenas JM. Colombia-Venezuela. Agenda común para el siglo XXI. Bogotá: Tercer Mundo Editores; 1999.

4. Gómez D. Análisis comparado de los sistemas de salud de la Región Andina y El Caribe. Rev Salud Pública 2005; 7:305-16.

5. Mesa-Lago C. Las reformas de salud en América Latina y el Caribe: su impacto en los principios de la seguridad social. Santiago de Chile: Comisión Económica para América Latina y el Caribe, Naciones Unidas; 2005.

6. Organismo Andino de Salud, Convenio Hipólito Unánue. Lineamientos estratégicos y líneas de acción. http://www.orasconhu.org/index.php?IDIO $\mathrm{MA}=\mathrm{SP} \&$ plantilla $=$ contenido\&ncategoria $1=101 \& \mathrm{n}$ contenido $=547$ (accedido el Oct/2006)

7. Docteur E, Oxley H. Health-care systems: lessons from the reform experience. Paris: Organisation for Economic Cooperation and Development; 2003. (OECD Health Working Papers, 9).

8. Organización Panamericana de la Salud. Análisis de las reformas del sector salud en los países de la Región Andina. Washington DC: Organización Panamericana de la Salud; 2003.
9. Le Bonniec Y. Sistemas de protección social comparados: Colombia, Brasil y México. Rev Salud Pública 2002; 4:203-39.

10. Organización Panamericana de la Salud. El derecho a la salud en las Américas. Washington DC: Organización Panamericana de la Salud; 1989. (Publicación Científica, 509).

11. Vargas I, Vásquez M, Jane E. Equidad y reformas de los sistemas de salud en Latinoamérica. Cad Saúde Pública 2002; 18:927-37.

12. World Bank. World development indicators database 2004. http://www.worldbank.org (accedido el May/2004).

13. Musgrove P, Zeramdini R, Carrin G. Basic patterns in national health expenditure. Bull World Health Organ 2002; 80:134-42.

14. Organización Panamericana de la Salud. Gasto nacional y financiamiento del sector salud en América Latina y el Caribe: desafíos para la década de los noventa. Washington DC: Organización Panamericana de la Salud; 2004. (Serie de Informes Técnicos, 30).

15. Organización Panamericana de la Salud. La salud en las Américas. Washington DC: Organización Panamericana de la Salud; 2002.

Recibido el 14/Jun/2006

Versión final presentada el 29/Ene/2007 Aprobado el 14/Feb/2007 